\title{
Identification of the Wheat Curl Mite as the Vector of Triticum mosaic virus
}

Dallas L. Seifers and T. J. Martin, Kansas State University, Agricultural Research Center-Hays, Hays, KS 676019228; Tom L. Harvey, Department of Entomology, Kansas State University, Manhattan 66506; John P. Fellers, USDA-ARS-PSERU, Dept. of Plant Pathology, Manhattan, KS 66506; and J. P. Michaud, Department of Entomology, Kansas State University, Manhattan 66506

\begin{abstract}
Seifers, D. L., Martin, T. J., Harvey, T. L., Fellers, J. P., and Michaud, J. P. 2009. Identification of the wheat curl mite as the vector of Triticum mosaic virus. Plant Dis. 93:25-29.

Triticum mosaic virus (TriMV) is a newly discovered virus found infecting wheat (Triticum aestivum) in Kansas. This study was conducted to determine if the wheat curl mite (WCM, Aceria tosichella) and the bird cherry oat aphid (Rhopalosiphum padi) could transmit TriMV. Using different sources of WCM and two different isolates of TriMV, we were able to show the WCM is the vector of TriMV. Field analysis by enzyme-linked immunosorbent assay (ELISA) demonstrated natural infection patterns of wheat infected with TriMV, Wheat streak mosaic virus (WSMV), or both TriMV and WSMV, putatively infected by viruliferous WCM from a volunteer source growing adjacent to the wheat. Moreover, by single WCM transfers using WCM obtained from different wheat plants naturally infected with TriMV and WSMV and naturally infested with WCM, we showed that these WCM also transmitted TriMV only to wheat or transmitted both TriMV and WSMV to wheat. The infection rates of wheat with TriMV only using WCM transmission was low in both laboratory and field analyses. However, field analyses by ELISA showed that levels of infection of wheat by both TriMV and WSMV were high. No transmission of TriMV to wheat by $R$. padi occurred in our studies.
\end{abstract}

In 2006, a new pathogen was isolated from wheat (Triticum aestivum L.) with virus-like symptoms in Kansas (10). The pathogen was mechanically transmissible and associated with a $35-\mathrm{kDa}$ protein when analyzed by sodium dodecyl sulfate polyacrylamide gel electrophoresis, and the antiserum raised to this protein preferentially reacted to it in enzyme-linked immunoassay (ELISA) and Western blot assay. In studies using electron microscopy, it was shown that the symptomatic plants infected by the pathogen were associated with virus-like particles, demonstrating that the pathogen was a virus.

The amino acid sequence of the $35-\mathrm{kDa}$ protein was determined using both timeof-flight mass spectrometry and DNA sequencing of cloned, reverse-transcribed viral RNA, and the results of these techniques showed that the virus was most closely related to Sugarcane streak mosaic virus (SCSMV) but only at the $49 \%$ level

Corresponding author: Dallas L. Seifers

E-mail: dseifers@ksu.edu

Contribution No. 08-353-J from the Kansas Agricultural Experiment Station.

Research in Kansas was partially funded by a grant from the Kansas Wheat Commission.

Accepted for publication 15 September 2008.

doi:10.1094/PDIS-93-1-0025

(C) 2009 The American Phytopathological Society
(10). Thus, the wheat-infecting virus was shown to not have been previously identified and the name Triticum mosaic virus (TriMV) was proposed.

During 2006, wheat plants infected with TriMV were not geographically localized, but were identified in several different locations in Kansas (10). The wheat was singly infected with TriMV, and also many wheat samples were infected with both TriMV and Wheat streak mosaic virus (WSMV). These plants were often infested with what appeared to be wheat curl mites (WCM, Aceria tosichella Keifer), and some samples had bird cherry oat aphids (BCOA, Rhopalosiphum padi (L.)) on them. Because of these observations, we investigated the possibility that the WCM or BCOA might transmit TriMV to wheat. We report here results demonstrating transmission of TriMV from wheat to wheat by the WCM.

\section{MATERIALS AND METHODS}

Virus source and maintenance. The U06-123 isolate of TriMV was isolated from KS06HW79 wheat in 2006 at the Kansas Agricultural Research Center-Hays (KSU-ARCH), Hays, KS (10). The Sidney 81 isolate of WSMV was collected at Sidney, NE, in 1981 and was obtained from W. G. Langenberg (11). The U07-431 isolates of TriMV and WSMV were obtained from a naturally infected volunteer wheat plant (unknown cultivar) growing in Ellis County, KS, in 2007 that, when analyzed by ELISA, tested doubly infected with both viruses. A pure isolate of each virus was obtained by inoculating mechanically 'Gallatin' barley (Hordeum vulgare L.) and 'Oh28' maize (Zea mays L.) with an extract prepared from plant U07-431. Oh28 maize is susceptible to infection with WSMV but not TriMV, and Gallatin barley is susceptible to infection with TriMV but not WSMV (D. L. Seifers, unpublished). Systemically infected barley or maize tissue was then used to prepare inoculum to inoculate wheat cv. Tomahawk. Systemically infected wheat was then used as the inoculum source for propagation of these isolates using the leaf-rub technique described previously (9). Following inoculation, the plants were held in a greenhouse under natural lighting.

Antiserum sources. Anti-TriMV serum was used as a whole serum at a 1:4,000 ( $\mathrm{vol} / \mathrm{vol})$ dilution prepared from a $1 \mathrm{mg}$ protein $/ \mathrm{ml}$ stock solution and was raised as described previously (10). Anti-WSMV serum (1:1,000 vol/vol) (6) and antiserum to the High Plains virus $(1: 1,000 \mathrm{vol} / \mathrm{vol})$ (HPV-WO) (4) were prepared or obtained as described previously, and working dilutions were made from stocks adjusted to 1 $\mathrm{mg}$ protein $/ \mathrm{ml}$.

Indirect ELISA. The procedure used was conducted as described previously (10). Antiserum concentrations were used as described above. Goat anti-rabbit antibody/alkaline phosphatase conjugate was used at a 1:3,000 vol/vol (Southern Biotechnology Associates, Birmingham, AL). Absorbance was measured at $405 \mathrm{~nm}$ using a Titertek Multiscan plate reader (Flow Laboratories, Inc., McLean, VA). Absorbance values were arbitrarily considered positive if they were twice those of the equivalent mock-inoculated control.

Source and maintenance of WCM and infestation of plants. The WCM used were from Ellis County, KS, and previously transmitted WSMV and HPV (7). Aviruliferous WCM (AvWCM) were obtained by raising adults from eggs and increasing them on caged Tomahawk wheat as described (7). This source of AvWCM was tested by ELISA, as described above, for the presence of WSMV and HPV before use in other experiments. Identifications of WCM were confirmed by J. W. Amrine (West Virginia University, Morgantown, WV).

Infectivity assays. Tomahawk wheat was inoculated mechanically (finger-rub) 
when at the single-leaf stage with 1:10 (wt/vol) extracts prepared from appropriate infected wheat sources as described above. Because the inoculated wheat plants were grown in large metal flats (as described above), they could not be caged (7). Following inoculation, the plants were held in a greenhouse under natural lighting, and symptom ratings were done at 14 to 21 days postinoculation (DPI).

WCM transmission of TriMV. Experiment 1. Ten Tomahawk wheat plants (grown in $2.4 \times 16.5 \mathrm{~cm}$ plastic planting cones; Stuewe \& Sons, Corvallis, OR) infected with the U06-123 isolate of TriMV were infested for $48 \mathrm{~h}$ with AvWCM by transfer from healthy Tomahawk wheat using a hair clip to attach a leaf piece (testing negative in ELISA and not infective in backassay) to the plants, and then each plant was covered in a cage (10). The AvWCM-infested wheat was then held at 20 to $22^{\circ} \mathrm{C}$ on a lab bench for $48 \mathrm{~h}$. Then the clipped tissue was removed and the infested leaves from the infected plants were bulked, cut into $1-\mathrm{cm}$ sections, and a single piece clipped to each of 25 Tomahawk and 25 RonL wheat plants (two-leaf stage). The plants were held at $18^{\circ} \mathrm{C}$ in a growth chamber with $8 \mathrm{~h}$ of fluorescent light $\left(250 \mu \mathrm{E} \cdot \mathrm{s}^{-1} \cdot \mathrm{m}^{-2}\right)$ for 3 weeks, and then each plant was rated for the presence of symptoms. Plants were held at $18^{\circ} \mathrm{C}$ because RonL is temperaturesensitive for resistance to WSMV (12). No attempt was made to determine the number of WCM on the leaf pieces. After 3 weeks, a leaf piece of the youngest leaf of each plant was analyzed by ELISA (as described above) using antiserum raised to TriMV, WSMV, and HPV. A leaf piece from individual plants testing positive in this analysis was harvested, and a 1:10 wt/vol extract was used to inoculate Tomahawk wheat assay plants. Leaf tissue was bulked from five plants from the aviruliferous control. At the same time, tissue from the TriMV and WSMV maintenance cultures was used to prepare equivalent extracts, each used separately to inoculate wheat plants in the assay. These assay plants were then held at $24^{\circ} \mathrm{C}$ for 3 weeks in a chamber with lighting conditions as described above.

For experiment 1, a second WCM transmission was done using WCM increased on six Tomahawk plants infested in the first part of the experiment, of which two were infected with TriMV, two were not infected, and two were from the AvWCM colony. Leaf pieces of varying sizes were taken from the six plants and were used to infest Tomahawk wheat (as described above). After $24 \mathrm{~h}$ on a bench top at 20 to $22^{\circ} \mathrm{C}$, the infested plants were moved to a greenhouse $\left(20\right.$ to $\left.27^{\circ} \mathrm{C}\right)$ under natural lighting conditions for 21 days and then rated for symptoms. Then tissue from the fourth leaf of each plant was used to inoculate Tomahawk wheat infectivity assay plants (as described above). The assay plants were held in the same greenhouse for 21 days, rated for symptoms, and then each plant was analyzed by ELISA (as described above).

Experiment 2. Twenty symptomatic Tomahawk plants infected with the U06123 isolate of TriMV were infested with AvWCM for $48 \mathrm{~h}$ (as described above). The infested leaves were bulked, and $1-\mathrm{cm}$ sections were used to infest 100 Tomahawk wheat plants as described above. The plants were held for 21 days in a greenhouse under natural lighting with a temperature range of 18 to $29^{\circ} \mathrm{C}$. Then the plants were rated for symptoms, and leaf three of each plant was analyzed by ELISA (as described above). Tissue from the third or fourth leaf from infected plants was used to mechanically inoculate Tomahawk wheat as described above, and the plants were held in a greenhouse under natural lighting for 21 days at 21 to $29^{\circ} \mathrm{C}$. At 21 DPI, the third leaf of each plant was analyzed by ELISA (as described above).

Experiment 3. One Tomahawk wheat plant infested with AvWCM was clipped (as described above) to three plants infected with either the U07-431 TriMV or U07-431 WSMV isolate on a lab bench for $48 \mathrm{~h}$ at room temperature $\left(20\right.$ to $\left.22^{\circ} \mathrm{C}\right)$. Then one virus-infected/WCM-infested plant was used to infest three Tomahawk wheat plants (two- to three-leaf stage) for $72 \mathrm{~h}$ at room temperature $\left(20\right.$ to $22^{\circ} \mathrm{C}$ ), so that 30 plants were infested for each virus. After $72 \mathrm{~h}$, each of the 60 plants was viewed under a microscope $(\times 15)$ to verify that each was infested with WCM and then held in a greenhouse under natural light. After 7 days, each infested plant was treated with Furadan 4EC (Carbofuran) to kill the WCM (7). The plants were held in a greenhouse under natural lighting at 22 to $30^{\circ} \mathrm{C}$. After 28 days, the plants were rated for symptoms, and leaf three of each plant was analyzed by ELISA (as described above). Because some plants died, each virus treatment did not have 30 plants for the analysis.

Leaf tissue from the seven Tomahawk wheat plants infected with U07-431 isolate of TriMV and six of those infected with the U07-431 isolate of WSMV in experiment 3 were used separately to inoculate Tomahawk wheat at the single-leaf stage (as described above). The inoculated plants were held in a greenhouse under natural lighting for 21 days at 19 to $30^{\circ} \mathrm{C}$. At 21 DPI, the third leaf of each plant was analyzed by ELISA (as described above).

Natural infection of wheat with TriMV and WSMV. Samples from a field of ' 2137 ' wheat in Ellis County, whose north edge was $7 \mathrm{~m}$ south of volunteer wheat (unidentified cultivar) having large numbers of plants with virus-like symptoms, were analyzed by ELISA for infection with TriMV, WSMV, and the HPV in April of 2007. Six rows, each $50 \mathrm{~m}$ long, were sampled at the location. Rows 1 and 2 were in the volunteer wheat, and rows 3 , 4,5 , and 6 were in the 2137 cultivar. Row 1 was at the south edge of the volunteer wheat; row $2,1 \mathrm{~m}$ north of row 1 ; row 3 , $10 \mathrm{~m}$ south of row 1 ; row $4,105 \mathrm{~m}$ south of row 3 ; row $5,105 \mathrm{~m}$ south of row 4 ; and row $6,105 \mathrm{~m}$ south of row 5 . Rows 1 and 3 were sampled on 3 April 2007, rows 4, 5, and 6 were sampled on 19 April 2007, and row 2 was sampled on 29 April 2007. The difference in sampling dates for rows 3,4 , 5 , and 6 in the 2137 wheat was because of weather conditions and, row 2 in the volunteer wheat was sampled later to locate fresh plants infected with both TriMV and WSMV and also heavily infested with WCM. In rows 1, 3, 4, 5, and 6, 25 symptomatic wheat plants were dug up approximately $2 \mathrm{~m}$ apart. In row 2,50 wheat plants were dug up at approximately $1 \mathrm{~m}$ apart. Each plant was placed in a selfsealing plastic bag, and this bag was then placed in a single larger plastic bag so that all bagged wheat plants were held in the larger bag. At each sampling date, the collected plants were taken to the lab, individual tillers were marked with numbered tape, and a piece of leaf tissue was taken from the marked tiller and placed in a like-numbered 1.5-ml microfuge tube. The leaf pieces were then weighed and immediately processed for analysis by ELISA (as described above).

Single WCM transfers from wheat naturally infected with both TriMV and WSMV. Plants U07-431, U07-439, and U07-440 (all testing positive for infection by both TriMV and WSMV) had 100 mites from each plant (adults and late-stage nymphs showing movement) transferred individually to separate Tomahawk wheat plants at the two-leaf stage (7). Transfer of the 100 mites from each plant occurred on three consecutive days. Each of the 300 infested plants was covered with a cage before and after transfer (7). The infested plants were held in a greenhouse under natural lighting. After 21 days, a portion of leaf three of each plant was analyzed by ELISA (as described above). WCM were not removed prior to analysis by ELISA.

BCOA. The BCOA colony was established from naturally infested Tomahawk wheat growing in a greenhouse at KSUARCH in January 2007. Apterous adults were transferred individually to 10 Tomahawk seedlings growing in plastic cones $(2.5 \mathrm{~cm}$ diameter $\times 16.5 \mathrm{~cm}$; Stuewe \& Sons, Corvallis, OR). After $24 \mathrm{~h}$, first instar nymphs were transferred individually with a fine camel hair brush to 20 seedlings, covered with cages, and held in a chamber (Percival model I-36VL) set to $22^{\circ} \mathrm{C}$ and a $16: 8$ (light:dark) photoperiod.

Nymphs were brushed off the plants onto a flat of wheat at the two-leaf stage. The flat of infested wheat was held in a chamber under the same conditions (as described above). New flats of wheat seed- 
lings were infested at approximately 1week intervals in the same manner.

TriMV transmission assay using BCOA. Ten first instar nymphs of $R$. padi were transferred as described above to each of 20 Tomahawk wheat plants infected with the U06-123 isolate of TriMV, covered with cages, and held in a chamber at $22^{\circ} \mathrm{C}$ and a 16:8 (light:dark) photoperiod. One week later, two to three $R$. padi alatae were transferred from the infected plants to each of 20 virus-free Tomahawk wheat plants as described above. Controls included 20 healthy Tomahawk wheat plants infested with two to three alatae from the virus-free colony, 20 virus-free, noninfested Tomahawk wheat plants, and 20 Tomahawk wheat plants inoculated with TriMV.

One week following infestation, the cages were removed and the plants exposed to a Vapona Pest Strip in a chamber for $2 \mathrm{~h}$. One week later, each plant was rated for symptoms, and the newest leaf of each plant was analyzed by ELISA. The experiment was performed twice.

\section{RESULTS}

WCM acquisition of TriMV using the Ellis County source of WCM. The WCM transmitted TriMV to wheat in each of the three experiments (Table 1), although the numbers of infected wheat plants were low. Two of 25 Tomahawk and one of 25 RonL plants, six of 100 Tomahawk plants, and seven of 29 Tomahawk plants were infected with TriMV in experiments 1,2 , and 3 , respectively. In contrast, 28 of 28 plants were infected with WSMV in experiment 3 (Table 1). Extracts from each of the plants infected with TriMV or WSMV were infective, and each contained antigens that reacted only with their homologous antiserum when analyzed by ELISA (data not shown).

The second transfer of WCM in experiment 1 from two plants infected with TriMV and infested with WCM resulted in two of 39 plants becoming infected using WCM from one plant and 0 of 14 plants infected using WCM from the second plant (data not shown). Antigens in the extracts from the two plants infected in the second cycle of transmission reacted only with TriMV antiserum in ELISA (data not shown).

Natural infection of wheat with TriMV and WSMV. The estimated per- centage of symptomatic plants decreased with distance from the volunteer wheat and ranged from $\sim 100 \%$ in row 3 to $\sim 5 \%$ in row 6 (Table 2). In row 1 of volunteer wheat, none of the 25 plants sampled were infected with TriMV, two were infected with WSMV only, and 23 were infected with both viruses. In row 2 of volunteer wheat, all 50 plants sampled were infected with both viruses. Wheat plants infected with WSMV alone and mixed infections of TriMV and WSMV were detected in all four rows of the cultivated wheat tested, although mixed infections were more common, $67 \%$ of the plants versus $22 \%$ of the plants infected with WSMV alone. Only $11 \%$ of the plants tested were infected with TriMV only, and they were found only in rows 4 and 5 . None of the plants tested were infected with HPV.

Single WCM transfers from wheat naturally infected with both TriMV and WSMV. The transfer of single WCM from plants U07-431, U07-439, and U07-440 that were infected with both viruses resulted in the transmission of TriMV or WSMV alone, or both TriMV and WSMV. Also, many of the single WCM did not transmit either of the viruses (Table 3). Successful colonization of the assay plants was indicated by WCM on individual wheat plants after 3 weeks, and was 59 of 100,63 of 100, and 44 of 100 for source plants U07-431, U07-439, and U07-440, respectively. Percentage values for infection were based only on the numbers of plants that were actually infested. A majority $(61 \%)$ of the single mite transfers from all three source plants resulted in the transmission of WSMV alone (101 of the 166 plants), whereas only four of the 166 mites transmitted TriMV alone (Table 3). WSMV may facilitate the transmission of TriMV, since 31 of the 166 mites transmitted both viruses. Thirty of the 166 mites did not transmit either virus. None of the plants that did not develop a WCM infestation tested positive by ELISA for either of the viruses, and HPV was not detected in any plant.

Mites increased from single mite infestation on two assay plants originating from the source plant U07-439, one assay plant infested with a mite from the source plant U07-440, and one assay plant infested with a mite from the source plant U07-431 were verified as WCM. One of the two plants infested with a WCM transferred from plant U07-439 was not infected and the other was infected with WSMV, and the two plants infested with WCM originating from source plants U07-439 and U07-440 were each infected with both TriMV and WSMV.

Tomahawk wheat plants infested with BCOA did not develop symptoms, and extracts from these plants did not react to TriMV antibodies in ELISA. Controls consisting of Tomahawk wheat plants mechanically inoculated by TriMV were symptomatic and contained antigens that reacted to only TriMV antibodies in ELISA (data not shown).

\section{DISCUSSION}

The TriMV nucleic acid sequence is most closely related to SCSMV (10), a

Table 2. Estimated percentage of symptomatic volunteer wheat plants (rows 1 and 2) and symptomatic 2137 cultivated wheat (rows 3 to 6) growing in adjacent fields in Ellis County, KS, in 2007, and the numbers of plants infected with Triticum mosaic virus (TriMV), Wheat streak mosaic virus (WSMV), or both TriMV and WSMV as determined by enzyme-linked immunosorbent assay

\begin{tabular}{lccccc}
\hline & \multirow{2}{*}{$\begin{array}{c}\text { Distance }(m) \\
\text { from row 1 } \\
\text { volunteer wheat }\end{array}$} & \multirow{2}{*}{$\begin{array}{c}\text { Estimated }^{\mathbf{x}} \\
\text { percent infection }\end{array}$} & 100 & \multicolumn{3}{c}{ Virus or viruses $^{\mathbf{y}}$} \\
\cline { 4 - 6 } Row & 0 & 100 & $0 / 25^{z}$ & $2 / 25$ & $23 / 25$ \\
1 & 1 & 100 & $0 / 50$ & $0 / 50$ & $50 / 50$ \\
2 & 11 & 85 & $0 / 25$ & $4 / 25$ & $21 / 25$ \\
3 & 116 & $6 / 25$ & $7 / 25$ & $12 / 25$ \\
4 & 221 & 5 & $5 / 25$ & $5 / 25$ & $15 / 25$ \\
5 & 336 & $0 / 25$ & $6 / 25$ & $19 / 25$ \\
6 & 5 & & & \\
\hline
\end{tabular}

${ }^{\mathrm{x}}$ Estimated percent infection is for plants in rows 1 to 6 .

${ }^{y}$ TriMV, WSMV, or TriMV/WSMV indicates the antiserum used to determine the virus or viruses infecting the plants in each row.

${ }^{\mathrm{z}}$ Numerator indicates number of plants infected and denominator indicates total number of plants tested in each row.

Table 1. Number of wheat plants that developed symptoms (NSP) following infestation with wheat curl mites that were previously given an acquisition feeding period on Tomahawk wheat infected with Triticum mosaic virus ${ }^{\mathrm{x}}$ (TriMV) or Wheat streak mosaic virus (WSMV)

\begin{tabular}{|c|c|c|c|c|c|c|c|c|}
\hline \multicolumn{9}{|c|}{ Experiment } \\
\hline \multicolumn{3}{|c|}{1} & \multicolumn{3}{|c|}{2} & \multicolumn{3}{|c|}{3} \\
\hline Cultivar & Virus & NSP & Cultivar & Virus & NSP & Cultivar & Virus & NSP \\
\hline Tomahawk & TriMV & $2 / 25^{z}$ & Tomahawk & TriMV & $6 / 100$ & Tomahawk & TriMV & $7 / 29$ \\
\hline RonL & TriMV & $1 / 25$ & & & & Tomahawk & WSMV & $28 / 28$ \\
\hline
\end{tabular}

${ }^{\mathrm{x}}$ The U06-123 isolate of TriMV was used in experiments 1 and 2, and the U07-431 isolate of TriMV was used in experiment 3.

${ }^{y}$ The U07-431 isolate of WSMV was used in experiment 3.

${ }^{\mathrm{z}}$ The numerator represents the number of symptomatic plants and the denominator the total number of plants infested. 
virus initially considered to be a member of the genus Tritimovirus in the family Potyviridae $(2,3)$, whose members have mite vectors (15). Because WCM were present on plants infected with TriMV alone or with TriMV and WSMV in 2006, we considered the possibility that the WCM might transmit TriMV. This was confirmed in our experiments, although the numbers of wheat plants infected with TriMV by WCM transmission were low (Table 1). We considered that the low numbers of infected plants might be a response of the TriMV isolate. This was based on other studies using the HPV where differences existed among HPV sources in efficiency of transmission by WCM (7). Thus, in the third experiment, we used a 2007 isolate of both TriMV and WSMV. We demonstrated for a third time that the same source of WCM could transmit the U07-431 isolate of TriMV, and the U07-431 isolate of WSMV. However, again low numbers of plants were infected with U07-431 isolate of TriMV, but all plants were infected with the U07431 isolate of WSMV in this experiment. The high numbers of plants infected with WSMV was expected because this source of WCM has transmitted both WSMV and HPV (7). It is possible that because both TriMV isolates were obtained in the same area of Kansas, the low transmission rate may be a response to the isolates in this area. Differential transmission of HPV isolates has been demonstrated for HPV (7). The use of isolates of TriMV from different geographical locations could not be used because, at present, TriMV has been identified only in Kansas (10).

Because of the importance of determining the vector of TriMV, infectivity assays were also conducted during the WCM transmission experiments for those plants considered infected with TriMV using WCM, to determine that they harbored infective particles of TriMV. This is because we have occasionally observed that when WCM are present in high numbers on wheat plants, such plants can occasionally give a false positive when analyzed by ELISA using anti-WSMV serum and are not infective in backassays (D. L. Seifers, unpublished). Such results were not ob- served in the present study using antiTriMV antiserum.

Although this source of the WCM transmitted TriMV from wheat to wheat, it is possible that the lower transmission rates with both TriMV isolates was a response of this colony of WCM. We showed in other studies that sources of WCM varied in transmission of the HPV isolates (7). It has been shown that physiological strains of the WCM exist that vary in their ability to transmit WSMV depending on if they were obtained from a source other than wheat (1). However, in our studies, the WCM source originated from wheat and was constantly maintained on wheat, removing that source of variation.

A decrease in the estimated infection levels occurred with distance from the source of volunteer wheat (Table 2). This is consistent with previous studies of WSMV and HPV infection of wheat by WCM $(8,16)$. At this study location, no other source of volunteer wheat existed for extended distances. Plants infected with TriMV only were identified, but as with our results from laboratory experiments, only in low numbers. These occurred in rows 4 and 5 and probably reflect a random sampling occurrence. We also identified wheat plants infected singly with WSMV in all rows of the 2137 wheat. Interestingly, we showed that, as for the volunteer wheat source, high numbers of the 2137 wheat plants were infected with both TriMV and WSMV, even in row 6, which was most distant from the volunteer wheat source and had a lower estimated percent infection. This might be accounted for because of the very large number of WCM found in the volunteer wheat source, so that of those WCM that infested the 2137 wheat plants in row 6 , many were transmitting both TriMV and WSMV. In contrast to our findings with TriMV and WSMV, the number of wheat plants infected with both HPV and WSMV immediately dropped $8 \mathrm{~m}$ past the first sampling point to just one plant (8). In another study, $36 \%$ of the wheat was infected with both WSMV and HPV (5).

Mites from the wheat source plants U07-431, U07-439, and U07-440 transmitted WSMV most often, followed by TriMV and WSMV, with single transmission of TriMV only four times (Table 3 ). This low level of transmission of TriMV by WCM is similar to our three transmission experiments using the U06-123 and the U07-431 TriMV isolates. Some of the mites from the single transfers were verified as WCM. Thus, because we transmitted both TriMV isolates by the Ellis County source of WCM, and the mites were verified as WCM from the above plants, it would be logical to assume that the mites that transmitted TriMV only were also WCM, although they were not among those mites verified. This is supported by the single infection of the wheat assay plants with WSMV and double infection with WSMV and TriMV of the other wheat assay plants, because the WCM is the only known vector of WSMV (14). In each transmission experiment, several mites from each source plant did not transmit any virus. Lack of transmission of WSMV by WCM obtained from wheat infected with WSMV has been shown to occur (1).

Interestingly, in the single WCM transmissions from source plants U07-431, U07-439, and U07-440, the numbers of wheat plants infected by both TriMV and WSMV was lower than we observed at the field location. This indicated that although the single WCM transfers from these three source plants verified the transmission of TriMV, either singly or with WSMV, it did not predict the levels of double infection observed in the field. This could be because many WCM that were viruliferous for both TriMV and WSMV initially infested the plants sampled in the field. Interestingly, the WCM is the vector of WSMV (14) and the HPV (8). Thus, with the transmission of TriMV, the WCM can transmit WSMV, a tritimovirus (15), TriMV, a virus suggested as a member of the family Potyviridae in a genus yet to be established (10), and the HPV, a likely member of a hitherto unclassified virus group (13).

In summary, we provided evidence using different WCM sources and two isolates of TriMV that supports the role of the WCM as a vector of TriMV. Field sampling demonstrated natural infection patterns of wheat infected with TriMV and

Table 3. Transmission efficiency of Triticum mosaic virus (TriMV), Wheat streak mosaic virus (WSMV), or both TriMV and WSMV by single wheat curl mites (WCM) transferred from three source wheat plants infected with both TriMV and WSMV

\begin{tabular}{lcccccccc}
\hline & \multicolumn{7}{c}{ Numbers and percentage of wheat plants infected by a given virus or NI } \\
\cline { 2 - 9 } Plant ID & TriMV & $\%$ & WSMV & \% & TriMV/WSMV & \% & NI & \% \\
\hline U07-431 & $4 / 59^{\mathrm{z}}$ & 6.8 & $29 / 59$ & 49.1 & $12 / 59$ & 20.3 & $14 / 59$ & 23.8 \\
U07-439 & $0 / 63$ & 0.0 & $43 / 63$ & 68.2 & $13 / 63$ & 20.7 & $7 / 63$ \\
U07-440 & $0 / 44$ & 0.0 & $29 / 44$ & 65.9 & $6 / 44$ & 13.7 & $9 / 44$ & 20.4 \\
\hline
\end{tabular}

${ }^{\mathrm{y}} \mathrm{NI}=$ not infected.

${ }^{\mathrm{z}}$ One hundred single mites were transferred from each source plant to 100 wheat seedlings. Successful infestation of the wheat seedlings with WCM was determined by the presence of viable mite colonies 3 weeks postinfestation and numbered 59/100, 63/100, and 44/100 from U07-431, U07-439, and U07440 , respectively. Only data from successfully infested plants are presented in the table. Numerator represents either the number of symptomatic wheat plants that tested positive by enzyme-linked immunosorbent assay (ELISA) for one virus (TriMV, WSMV) or both viruses (TriMV/WSMV), or the number of asymptomatic wheat plants that were infested with WCM but did not test positive by ELISA for either of the viruses (NI). Denominator represents the total number of the 100 starting wheat plants that were successfully infested by the individual transferred WCM. 
WSMV. Moreover, using single WCM transmission from wheat plants naturally infected with TriMV and WSMV and naturally infested with WCM, we showed that these WCM transmitted TriMV or WSMV only, or transmitted both TriMV and WSMV to wheat. The numbers of wheat plants infected with TriMV only were low. However, field sampling of wheat showed that levels of infection by both TriMV and WSMV were high. Additional studies are needed concerning the effect of WCM growth stage, length of acquisition feeding time, inoculation feeding time, and temperature during both acquisition and inoculation feeding times on transmission of TriMV. In addition, the interaction of different WCM sources and TriMV isolates on transmission of TriMV should be investigated both singly and in combination with WSMV.

\section{ACKNOWLEDGMENTS}

We thank Jeff Ackerman for his valuable assistance in ELISA, WCM transfers, and greenhouse studies during this investigation, Clayton Seaman for assistance with greenhouse studies, Jawahar Jyoti for his assistance in aphid experiments, and $\mathrm{J}$. W. Amrine for identification of the WCM.

\section{LITERATURE CITED}

1. del Rosaria, M. S. E., and Sill, W. H., Jr. 1965. Physiological strains of Aceria tulipae and their relationships to the transmission of wheat streak mosaic virus. Phytopathology 55:11681175 .

2. Hall, J. S., Adams, B., Parsons, T. J., French, R., Lane, L. C., and Jensen, S. G. 1998. Molecular cloning, sequencing and phylogenetic relationships of a new potyvirus: Sugarcane streak mosaic virus and a reevaluation of the classification of the Potyviridae. Mol. Phylogen. Evol. 10:323-332.

3. Hema, M., Joseph, J., Gopinath, K., Sreenivasulu, P., and Savithri, H. S. 1999. Molecular characterization and interviral relationships of a flexuous filamentous virus causing mosaic disease of sugarcane (Saccharum officinarum) in India. Arch. Virol. 144:479-490.

4. Louie, R., Seifers, D. L., and Bradfute, O. E. 2006. Isolation, transmission and purification of the High Plains virus. J. Virol. Methods 135:214-222.

5. Mahmood, T., Hein, G. L., and Jensen, S. G. 1998. Mixed infection of hard red winter wheat with High Plains virus and wheat streak mosaic virus from wheat curl mites in $\mathrm{Ne}$ braska. Plant Dis. 82:311-315.

6. Seifers, D. L. 1992. Partial characterization of a Colorado isolate of Agropyron mosaic virus. Plant Dis. 76:564-569.

7. Seifers, D. L., Harvey, T. L., Louie, R., Gordon, D. T., and Martin, T. J. 2002. Differential transmission of isolates of the High Plains virus by different sources of wheat curl mites. Plant Dis. 86:138-142.

8. Seifers, D. L., Harvey, T. L., Martin, T. J., and Jensen, S. G. 1997. Identification of the wheat curl mite as the vector of the High Plains virus of corn and wheat. Plant Dis. 81:1161-1166.

9. Seifers, D. L., and Martin, T. J. 1988. Correla- tion of low level of Wheat streak mosaic virus resistance in Triumph 64 wheat with low virus titer. Phytopathology 78:703-707.

10. Seifers, D. L., Martin, T. J., Harvey, T. L. Fellers, J. P., Stack, J. P., Ryba-White, M. Haber, S., Krokhin, O., Spicer, V., Lovat, N., Yamchuk, A., and Standing, K. G. 2008. Triticum mosaic virus: A new virus isolated from wheat in Kansas. Plant Dis. 92:808-817.

11. Seifers, D. L., Martin, T. J., Harvey, T. L., and Gill, B. S. 1995. Temperature sensitivity and efficacy of wheat streak mosaic virus resistance derived from Agropyron intermedium. Plant Dis. 79:1104-1106.

12. Seifers, D. L., Martin, T. J., Harvey, T. L. Haber, S., and Haley, S. D. 2006. Temperature sensitivity and efficacy of Wheat streak mosaic virus resistance derived from C0960293 wheat. Plant Dis. 90:623-628.

13. She, Y.-M., Seifers, D. L., Haber, S., Ens, W. and Standing, K. G. 2004. Characterization of the agent of 'High Plains Disease': Mass spectrometry determines the sequence of the disease-specific protein. J. Biol. Chem. 279:488494.

14. Slykhuis, J. T. 1955. Aceria tulipae Keifer (Acrina: Eriphidae) in relation to the spread of wheat streak mosaic. Phytopathology 45:116128.

15. Stenger, D. C., Hall, J. S., Choi, I.-R., and French, R. 1998. Phylogenetic relationships within the family Potyviridae: Wheat streak mosaic virus and brome streak mosaic virus are not members of the genus Rymovirus. Phytopathology 88:782-787.

16. Willis, W. G. 1981. The 1981 Wheat Streak Mosaic Epidemic in Kansas. Coop. Ext. Rep. Kansas State University, Manhattan. 